\title{
Nanostructured Polymeric Coatings Based on Chitosan and Dopamine-Modified Hyaluronic Acid for Biomedical Applications
}

\author{
Ana I. Neto, Ana C. Cibrão, Clara R. Correia, Rita R. Carvalho, Gisela M. Luz, \\ Gloria G. Ferrer, Gabriela Botelho, Catherine Picart, Natália M. Alves, * and João F. Mano
}

\begin{abstract}
In a marine environment, specific proteins are secreted by mussels and used as a bioglue to stick to a surface. These mussel proteins present an unusual amino acid 3,4-dihydroxyphenylalanine (known as DOPA). The outstanding adhesive properties of these materials in the sea harsh conditions have been attributed to the presence of the catechol groups present in DOPA. Inspired by the structure and composition of these adhesive proteins, we used dopamine-modified hyaluronic acid $(H A-D N)$ prepared by carbodiimide chemistry to form thin and surface-adherent dopamine films. This conjugate was characterized by distinct techniques, such as nuclear magnetic resonance and ultraviolet spectrophotometry. Multilayer films were developed based on chitosan and HA-DN to form polymeric coatings using the layer-by-layer methodology. The nanostructured films formation was monitored by quartz crystal microbalance. The film surface was characterized by atomic force microscopy and scanning electron microscopy. Water contact angle measurements were also conducted. The adhesion properties were analyzed showing that the nanostructured films with dopamine promote an improved adhesion. In vitro tests showed an enhanced cell adhesion, proliferation and viability for the biomimetic films with catechol groups, demonstrating their potential to be used in distinct biomedical applications.
\end{abstract}

A. I. Neto, A. C. Cibrão, C. R. Correia, R. R. Carvalho,

G. M. Luz, N. M. Alves, J. F. Mano

3B's Research Group - Biomaterials, Biodegradables and Biomimetics

University of Minho

AvePark, 4806-90, Taipas, Guimarães, Portugal

E-mail: nalves@dep.uminho.pt

A. I. Neto, A. C. Cibrão, C. R. Correia, R. R. Carvalho,

G. M. Luz, N. M. Alves, J. F. Mano

ICVS/3B's PT Government Associate Laboratory

Braga/Guimarães, Portugal

G. G. Ferrer

Center for Biomaterials and Tissue Engineering

Universitat Politècnica de València

Camino de vera $\mathrm{s} / \mathrm{n}$

46022, València, Spain

DOI: $10.1002 / \mathrm{smll} .201303568$
G. G. Ferrer

Networking Research Center on Bioengineering

Biomaterials and Nanomedicine (CIBER-BBN)

València, Spain

G. Botelho

Center/Department of Chemistry

University of Minho

Campus de Gualtar

4710-057, Braga, Portugal

C. Picart

LMGP, UMR 5628 CNRS/INPG

3 Parvis Louis Néel 38016, Grenoble, France 


\section{Introduction}

It is well known that biomaterials interact with cells and tissues through their interfaces, so surface properties are determinant on cell behavior. ${ }^{[1]}$ In some situations it is also important that biomaterials adhere to tissues and organs. ${ }^{[2]}$ The development of adhesive substrates that would enhance cell attachment and proliferation and could also adhere to tissues would constitute a substantial breakthrough for tissue engineering and other biomedical applications. Mussel adhesive proteins (MAPs) found in marine mussels form outstandingly moisture- resistant adhesive bonds capable of surviving to the destructive conditions of the ocean. Such proteins provide the role of cement, which upon secretion become quickly solidified in wet environments via chemical crosslinking into an insoluble plaque, binding the animal to surfaces. ${ }^{[3]}$

It is known that MAPs contain considerable concentrations of an amino acid, 3,4-dihydroxy-L-phenylalanine (DOPA), which is formed by postranslational modification of tyrosine. A key aspect of DOPA and its analog dopamine (DN) is the ortho-dihydroxyphenyl (catechol) functional group, which forms strong bonds with various inorganic/ organic surfaces. ${ }^{[4]}$ Several authors have developed new adhesives trying to mimic the adhesion properties found in marine mussels. ${ }^{[5]}$ For example, Deming and co-workers synthesized simple co-polypeptides of L-lysine and DOPA with different compositions. ${ }^{[5 \mathrm{~b}]} \mathrm{Yu}$ et al. synthesized copolymers of DOPA and L-lysine that form moisture-resistant adhesive bonds with a variety of substrates (aluminum, steel, glass and plastics) when suitably oxidized. ${ }^{[5]}$ Anderson et al. also showed that the adhesion of DOPA/N5- (2-hydroxyethyl)L-glutamine films to mica and $\mathrm{TiO}_{2}$ surfaces increases with DOPA concentration. ${ }^{[5 \mathrm{~d}]}$ Lee et al. ${ }^{[5 \mathrm{e}]}$ developed new thermo-sensitive and injectable DOPA modified hyaluronic acid (HA)/Pluronic hydrogels and showed that these hydrogels exhibited excellent tissue-adhesion properties with superior in vivo gel stability.

MAPs are in fact a source of inspiration for the development of nanostructured materials. Some techniques, such as layer-by-layer (LbL) have been used in the production of such functional materials. LbL is a simple methodology that allows the possibility to coat surfaces through multiple deposition steps, with an ability to control the build-up of complex geometries at the nanometer length scale. This constitutes a great advantage in comparison to other thin film assembly techniques. Multilayer films were produced by LbL using chitosan (CHT) and dopamine modified hyaluronic acid (HA-DN) in the present work, in order to try to combine the adhesion properties found in marine mussels with a simple film production method and with the known advantages of CHT and HA for biomedical applications.

In fact, HA and CHT are very popular biomaterials that have been often to form nanostructured coatings, ${ }^{[6]} \mathrm{HA}$ is a polysaccharide that can be found in tissues and body fluids of vertebrates and also in some bacteria. ${ }^{[7]}$ It is a linear polymer and a naturally occurring non-sulfated glucosaminoglycan composed of D-N-acetylglucosamine and D-glucuronic acid. ${ }^{[5,8]} \mathrm{HA}$ is a negatively charged polyelctrolyte above its pKa $(\approx 2.9) .{ }^{[9]}$ Because of its excellent lubricity and bacterial inhibitory effect, HA is suitable for tissue engineering applications. ${ }^{[8,10]} \mathrm{CHT}$, due to the primary amines present in its structure, is a positively charged polyelectrolyte in acidic medium, with a $\mathrm{pKa} \approx 6.24$ It is non toxic, biodegradable and has an excellent biocompatibility. So, CHT is a great candidate for a series of biomedical applications. ${ }^{[11]}$

Several works have demonstrated the advantages of using the materials chosen in the present work in the biomedical field. For example, the surface of titanium, a common implant material for orthopaedic applications, has been modified with HA and CHT coatings, allowing to promote osteoblast functions. ${ }^{[10]}$ Also, Lee et al $^{[3]}$ have incorporated catechol groups (which are present in $\mathrm{DN}$ ) into coatings of distinct substrates and induced the reduction of silver ions to metallic silver when immersed in an aqueous metal salt solution, providing antibacterial properties to the coated surfaces.

There are only a few studies where LbL films inspired by MAPs were studied ${ }^{[3,12]}$ A recent work of Zhang et al. ${ }^{[12 b]}$ showed that multilayer films of dopamine-modified hyaluronic acid/chitosan (DHA/CHI) built on the surface of Ti-24Nb-2Zr (TNZ) alloy can improve osteoblast proliferation and biocompatibility for orthopedics applications, when compared with to original ones.

In this work, we hypothesize that the formation of multilayers films of conjugate HA-DN and CHT can produce surfaces with distinct properties, when compared with the conventional $\mathrm{CHT} / \mathrm{HA}$ ones. The greatest advantage that the current approach presents over other reported methods is that we try to combine an enhanced cell response with a significantly improved adhesive strength, whereas the other reported methods have only showed improvements on cell behaviour. The combination of this particular system could be extremely advantageous regarding biomedical applications.

The formation of CHT and HA-DN multilayer films by the LbL technique was investigated using quartz crystal microbalance with dissipation monitoring (QCM-D). The film surface was characterized by atomic force microscopy (AFM) and scanning electron microscopy (SEM). The wettability was analyzed by water contact angle measurements. The mechanical adhesive properties and the in vitro cell behavior were evaluated on such nanostructured films inspired on mussel adhesive proteins.

\section{Results and Discussion}

\subsection{HA-DN Conjugate Synthesis and Characterization}

In order to know if the synthesis of the conjugate was successful the product of the synthesis was analyzed by two techniques: NMR and UV spectroscopy.

The ${ }^{1} \mathrm{H}-\mathrm{NMR}$ spectrum of the unmodified hyaluronic acid is shown in Figure 1A and is consistent with the one found in literature, ${ }^{[13]}$ where the multiplets located at $\delta=$ $2.028 \mathrm{ppm}$ are associated with the protons of $\mathrm{N}-\mathrm{COCH} 3$ groups. Figure $1 \mathrm{~B}$ shows the ${ }^{1} \mathrm{H}-\mathrm{NMR}$ spectrum of dopamine. The multiplets centered at $\delta=2.876 \mathrm{ppm}$ and $\delta=3.227 \mathrm{ppm}$ 

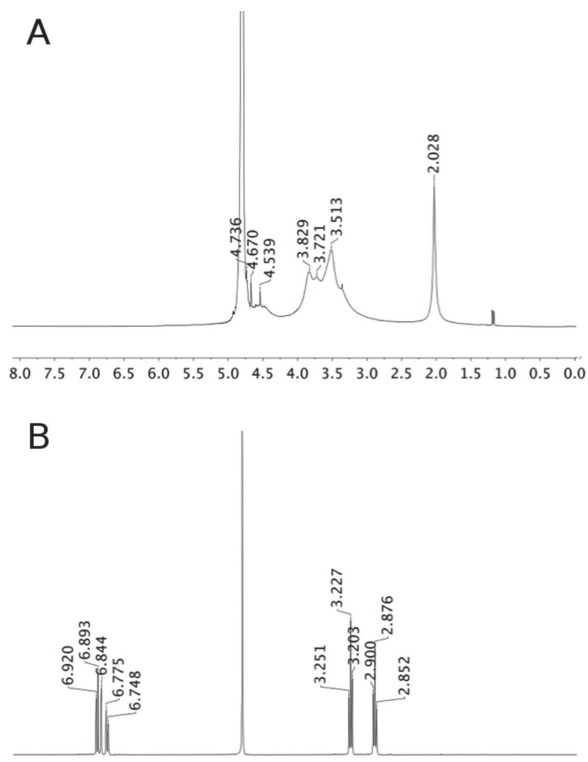

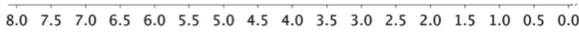
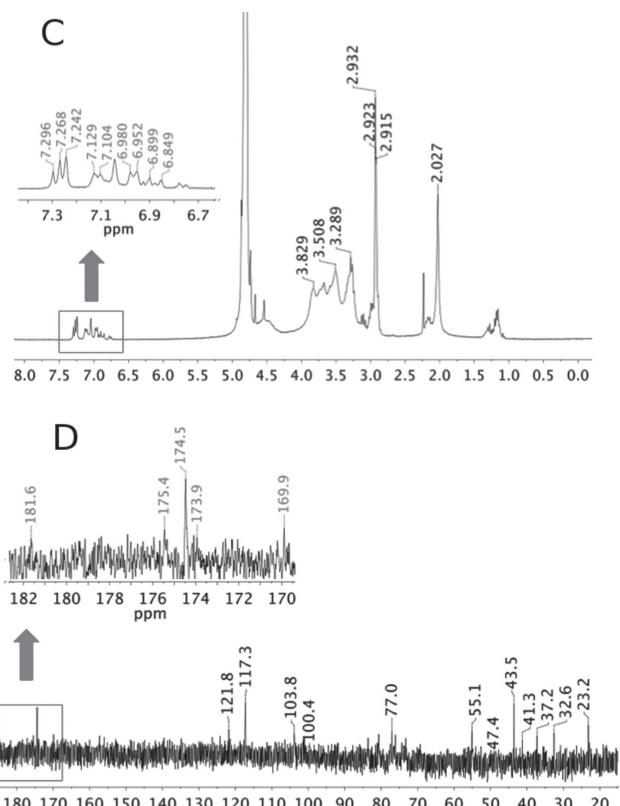

Figure 1. ${ }^{1} \mathrm{H}-\mathrm{NMR}$ spectra of A) hyaluronic acid (HA), B) dopamine and C) the synthesized conjugate (HA-DN) with an expanded view. ${ }^{13} \mathrm{C}-\mathrm{NMR}$ spectra of the conjugate with expanded view.

that can be observed in this Figure are associated with the protons of the aliphatic group, according to the literature. ${ }^{[14]}$ The region between $\delta=6.7 \mathrm{ppm}$ and $\delta=7.0 \mathrm{ppm}$ corresponds to the protons in orto and meta coupling position of the ring.

By using the experimental conditions previously described, the conjugate was obtained and characterized by ${ }^{1} \mathrm{H}-\mathrm{NMR}$ spectrum - see Figure $1 \mathrm{C}$. It is known that the new $\mathrm{N}-\mathrm{H}$ linkage formed in the conjugate could not be detected by ${ }^{1} \mathrm{H}-\mathrm{NMR}$ as this conjugate is only soluble in water and in this solvent the $\mathrm{NH}$ linkage interchange with water so no new signal appears. In order to overcome this limitation and using the conditions described in the experimental section, the conjugate was obtained and characterized by ${ }^{13} \mathrm{C}-\mathrm{NMR}$. The ${ }^{13} \mathrm{C}$-NMR spectrum of the unmodified hyaluronic acid is consistent with published results. ${ }^{[15]}$ According to the literature, ${ }^{[16]}$ the carbonyl chemical shift of HA should be between 173 and $174 \mathrm{ppm}$. The ${ }^{13} \mathrm{C}-\mathrm{NMR}$ new signal of around
175 ppm shown in Figure 1D confirms that the HA-DN conjugate was successfully synthesized.

The results obtained by UV-Vis spectroscopy are presented in Figure 2. It can be seen that, at approximately $280 \mathrm{~nm}$, one band appears for the HA-DN conjugate, being not observed in HA - see Figure 2A.

In fact, dopamine presents a band in the UV spectrum at this wavelength. ${ }^{[17]}$ These results indicate the presence of dopamine in the synthesized product in agreement with the NMR results. Several spectra of solutions with different concentrations of dopamine can be seen in Figure 2B; they all have an excitation band at approximately $280 \mathrm{~nm}$, similar to the conjugate band. Using the measured absorbance of the conjugate, obtained at $280 \mathrm{~nm}$, it was determined that the concentration of DN units in the HA-DN solution was $2.86 \times 10^{-4} \mathrm{M}$. Therefore, the degree of dopamine substitution in the synthesized conjugate is $11 \%$, being different than
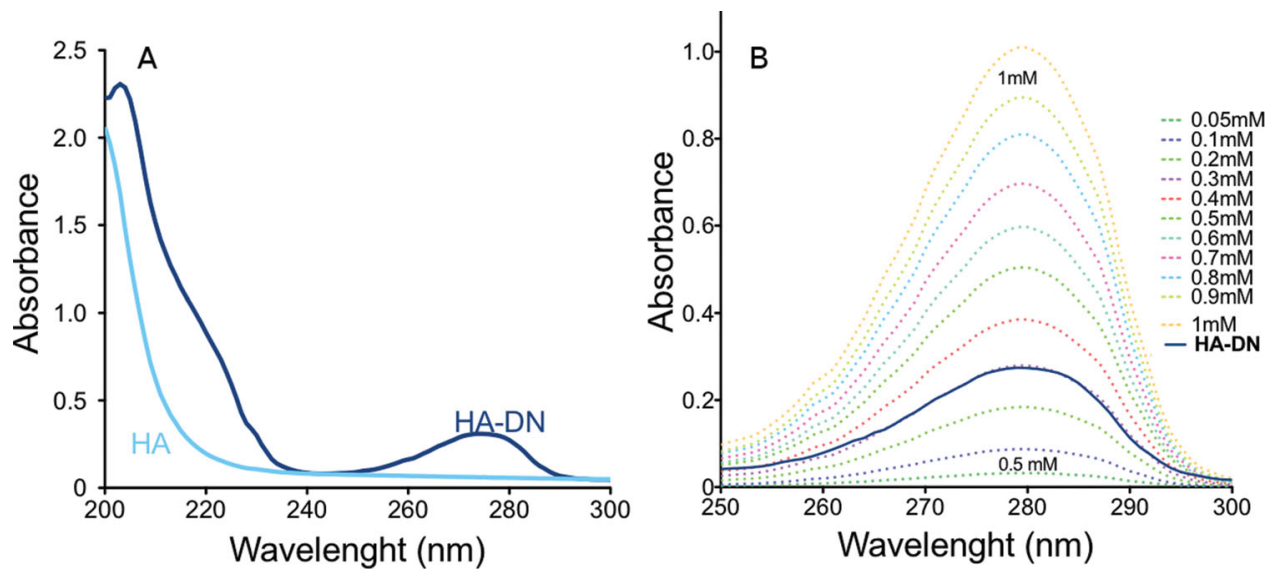

Figure 2. A) UV-Vis spectra of the conjugate (HA-DN) and the control (HA). B) Spectra of dopamine solutions with different concentrations; the spectrum of the conjugate is also shown for comparison. 

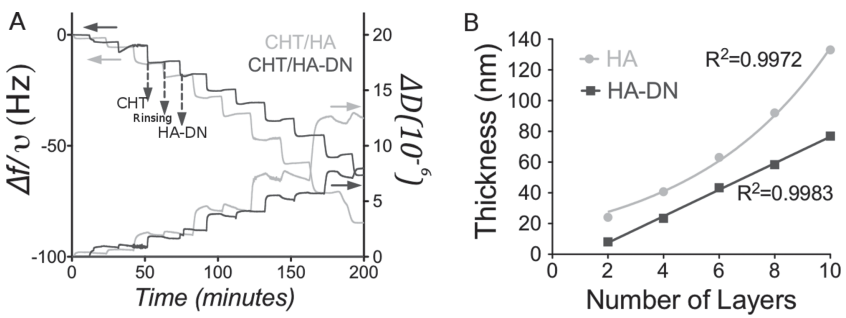

Figure 3. Build-up assemblies of chitosan (CHT), conjugate (HA-DN and hyaluronic acid (HA) up to 10 deposition layers in $0.15 \mathrm{M}$ of $\mathrm{NaCl}$ at $\mathrm{pH}$ 5.5. A) Quartz-crystal microbalance with dissipation monitoring $(Q C M-D)$ with normalized frequency $(\Delta f / v)$ and dissipation $(\Delta D)$ variations at the 7 th overtone as a function of the time. B) Cumulative thickness evolution of $\mathrm{CHT} / \mathrm{HA}-\mathrm{DN}$ and $\mathrm{CHT} / \mathrm{HA}$ polymeric films as a function of the number of deposition layers. Thickness measurements were estimated using a Voigt viscoelastic model.

the ones found in other works. ${ }^{[3,4 \mathrm{~b}, 5 \mathrm{e}]}$ However some conditions, such as the relative proportions of the reagents used and/or the time of the reaction, employed in the synthesis of HA-DN differ from the ones used in these studies.

\subsection{Build-up of CHT/ HA-DN Multilayer Films and Surface Characterization}

The development of LbL films containing materials inspired by MAPs is still very scarce. Lee et al. ${ }^{\left[{ }^{[3}\right.}$ were the first to propose this kind of films and successfully assembled HA-DN with poly(ethylenimine). The composition, structure and the crosslinking methodology lead to the development of $\mathrm{LbL}$ films with high strength. Charlot et al. ${ }^{[12 a]}$ prepared robust antimicrobial films on stainless steel based on the LbL deposition of polyelectrolytes that contained DOPA. A polycationic copolymer (N-methacrylated DOPA) with (2-(methacryloxy) ethyl trimethylammonium chloride) known as P(DOPA)co-P(DMAEMA+), was synthesized and co-deposited with precursors of silver nanoparticles as the first layer. Multilayer films obtained by alternating this nanoparticle-loaded polycationic copolymer with polystyrene sulfonate, a commercial polyanion, resulted in stainless steel with high antibacterial activity against Gramnegative E. coli bacteria. ${ }^{[12 a]}$

In the present work the formation of LbL films of CHT/HA-DN and CHT/HA was investigated in situ using QCM-D- see Figure 3A. For graphical simplification, the results in this figure present $\Delta f / v$ and $\Delta D$ as a function of time for the 7 th harmonic. It can be seen that the normalized frequency $\Delta f / v$ decreases with each CHT, HA-DN or HA injection, indicating that mass is added during each deposition step. When rising with $\mathrm{NaCl}$ solution after $\mathrm{CHT}$, HA-DN or HA adsorption, an increase in $\Delta f / v$ is observed due to the desorption of a small fraction of free polyelectrolyte. Regarding $\Delta D$, an increase is observed after each CHT, HA-DN or HA injection due to the viscoelastic nature of the adsorbed layers, typical of polymeric systems.

Figure $3 \mathrm{~A}$ also shows that $\Delta f / v$ and $\Delta D$ variations are higher for the CHT/HA film than for the CHT/HA-DN film. The lower $\Delta D$ variation of CHT/HA-DN (Figure $3 \mathrm{~A}$ ) indicates the formation of a thinner, more rigid and less waterrich film. Figure 3B shows that the $\mathrm{CHT} / \mathrm{HA}$ film presents an exponential film growth, which could explain the higher $f / v$ variation for this system, whereas the CHT/HA-DN film represents a linear trend line. Other authors have reported an exponential growth of polyelectrolyte films containing chitosan and hyaluronic acid, ${ }^{[18]}$ in agreement with our results for the control films. Two explanations for an exponential growth mechanism have been proposed in literature: one relies on the diffusion of polyelectrolyte "in" and "out" of the film during each bilayer step ${ }^{[18]}$ whereas the second one relies on the increase in film surface roughness as the film builds up ${ }^{[19]}$ In order to clarify the mechanism of the exponential growth, the surface roughness of the developed and control films was analysed by AFM- see Figure 4. In fact, it was found that the roughness of the $\mathrm{CHT} / \mathrm{HA}$ films is higher than the one of the CHT/HA-DN films, suggesting that the $\mathrm{CHT} / \mathrm{HA}$ interface is getting rougher and the surface area is increasing so that more CHT or HA can absorb. Figure 4 also shows the results of an additional characterization of the film surface by SEM and water contact angle measurements. The results for the control (glass slide) are also shown for the sake of comparison. The changes in the water contact angle and topography of the glass control provided evidence of the presence of the coatings. SEM and AFM results also showed
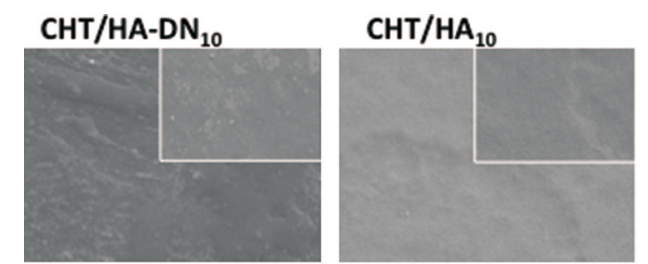

Control
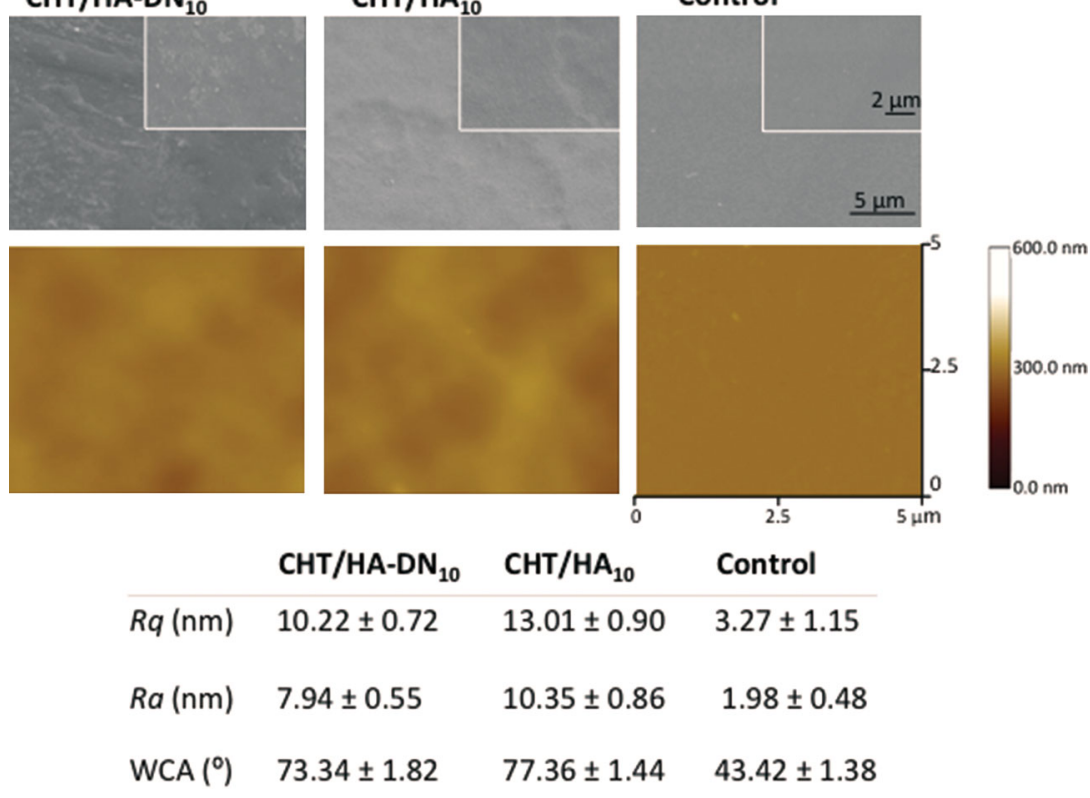

Figure 4. Representative images of scanning electron microscopy (SEM) and atomic force microscopy (AFM) of CHT/HA-DN and CHT/HA films with 10 bilayers. The images of the control (glass slide) are also shown for the sake of comparison. The root mean Square $\left(R_{\mathrm{q}}\right)$, average roughness $\left(R_{\mathrm{a}}\right)$ of the studied surfaces $(\mathrm{n}=3)$ and corresponding water contact angle (WCA, $\mathrm{n}=5,3 \mu \mathrm{L}$ drop volume) are indicated below the images. 
the rougher surface of the coatings when compared with the control surface. Moreover, it was found that the water contact angle values of the CHT/HA-DN and CHT/HA films are similar, although the CHT/HA-DN value is slightly lower (around $73^{\circ}$ ) than the one of the CHT/HA films (around $77^{\circ}$ ). Water contact angles of nearly $50^{\circ}$ were reported for polydopamine coated glass substrates, ${ }^{[20]}$ which are significantly lower than the ones measured for the developed CHT/ HA-DN coatings. In our case, as the dopamine fraction in the films is small, the corresponding contact angle is also small.

Further information on the viscoelastic properties of the formed films were obtained by applying a Voigt based model. ${ }^{[21]}$ The data of Figure $3 \mathrm{~B}$ were fitted according to this model. By assuming a fluid density of $1000 \mathrm{~kg} . \mathrm{m}^{-3}$, a fluid viscosity of 1 m.Pa.s and a layer density of $1100 \mathrm{~kg} \cdot \mathrm{m}^{-3}$ it was possible to estimate the thickness as a function of the number of layers during the build-up of the film - see Figure 3B. Lee and co-workers, measured the thickness of LbL films based on poly(ethylenimine) and HA-DN with 20 bilayers and they obtained a thickness of approximately $45 \mathrm{~nm} .^{[3]}$ We found that the thickness of the CHT/HA-DN film with 10 layers is approximately $75 \mathrm{~nm}$ and for CHT/HA is $130 \mathrm{~nm}$ at $\mathrm{pH}$ 5.5. It can also be seen that the thickness of the HA-DN film is lower than the one of HA film, as expected taking into account the $\Delta D$ variation and the different growth, as already discussed earlier. It must be noted that the estimated values of thickness are based on the assumption that the adsorbed layer has an uniform thickness, a necessary requirement for the used viscoelastic model. ${ }^{[22]}$ The thickness values estimated by fitting of the QCM-D data were further confirmed by ellipsometry measurements. Films built in the same conditions as the ones used in the QCM-D experiments were analysed by ellipsometry. The thickness values were $94.06 \pm 4.42 \mathrm{~nm}$ and $124.42 \pm 2.50 \mathrm{~nm}$ for the CHT/HA-DN and the CHT/HA films, respectively, which are in agreement with the QCM-D results.

It should be pointed that the conjugation of dopamine to the $\mathrm{COOH}$ groups of HA should affect its pKa. However, as the degree of DOPA substitution in the prepared conjugate was just $11 \%$ as measured by UV-spectrophotometry, and given the neutral nature of the molecule, this change should not be so significant. In fact, the QCM data showed that is possible to build the films by $\mathrm{LbL}$ at $\mathrm{pH} 5.5$, meaning that HA-DN is still negatively charged.

\subsection{Adhesion Properties of the CHT/HA-DN Films}

The adhesion strength of multilayer and nanostructured films using the LbL technology was studied for the first time. The adhesion of CHT/HA-DN and CHT/HA (negative control) films to a glass substrate was evaluated using a universal mechanical testing machine, according to the standard procedure ASTM D1002. Figure 5A presents representative loadextension curves of both coatings, showing that the final displacement of CHT/HA-DN is higher, which indicates a higher adhesive strength compared with the control, CHT/HA. The adhesive strength results of the multilayer films are shown in Figure 5B. In fact, it can be seen in Figure $5 \mathrm{~B}$ that the adhesive strength of CHT/HA-DN is $2.32 \pm 2.20 \mathrm{MPa}$ and for $\mathrm{CHT} / \mathrm{HA}$ is $0.75 \pm 0.14 \mathrm{MPa}$. It is most likely that the increase in the adhesion force is due to the strong adhesion force of the catechol moieties in the modified HA. It should be mentioned that adhesion tests were also performed with films stained with eosin, which specifically stains the CHT component of the films. It was observed that, after plate detachment, both plates were stained, as shown in the representative image of the glass plates at the rupture point that is included in Figure 5. This is an indication that break occurred somewhere between the layers of the film.

Yamada et al. used the tyrosinase-catalyzed reactions of dopamine to try to confer adhesive properties to semidilute solutions of CHT and analysed their adhesive strength using glass as the substrate. ${ }^{[23]}$ Adhesive shear strengths of over $0.4 \mathrm{MPa}$ were observed for these modified CHT samples, while control CHT solutions conferred no adhesive strength. These values are about one order of magnitude lower than the results of this work.

$\mathrm{Yu}$ et al. measured the adhesive strength of DOPA and L-lysine copolymers using the same mechanical testing protocol adopted in this work and found that they formed moisture-resistant adhesive bonds with a variety of substrates (aluminum, steel, glass and plastics). ${ }^{[5]}$ They obtained values of adhesive strength were between 0.5 and $4 \mathrm{MPa}$, depending on the substrate and the oxidation conditions. Distinct oxidizing agents, air $\left(\mathrm{O}_{2}\right), \mathrm{NaIO}_{4}, \mathrm{H}_{2} \mathrm{O}_{2}$ and mushroom tyrosinase were used in that work. ${ }^{[5]}$ The authors found that successful adhesiveness was dependent on oxidation conditions and that the choice of oxidant was partially substrate-specific. In our case, the measured adhesive properties presented in Figure 5 correspond to un-oxidized films, and high adhesion strength was found for the films containing dopamine without the need of using oxidizing agents. Nevertheless, mechanical adhesion tests were also performed using sodium periodate as the oxidizing agent, which was found in literature to be an effective oxidizing agent of the catechol groups. ${ }^{[24]}$ For the used sodium periodate concentration
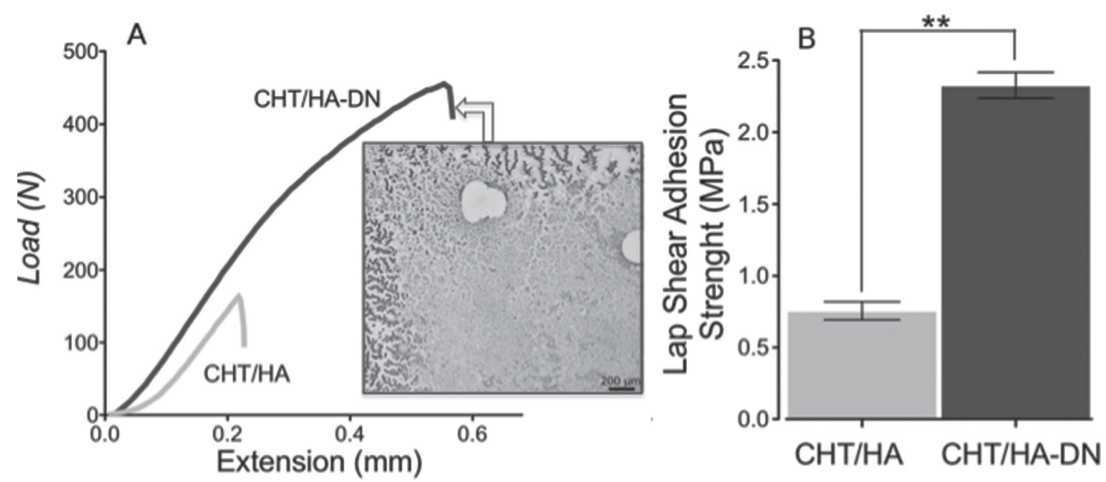

Figure 5. A) Force vs Displacement curves of $\mathrm{CHT} / \mathrm{HA}$ and $\mathrm{CHT} / \mathrm{HA}-\mathrm{DN}$ films. B) Adhesive strength of CHT/HA and CHT/HA-DN films between glass slides. Data are means SD $(n=5$; $\star \star * ~ p<0.01)$. Representative image of the glass slides at rupture point. 
( $5 \mathrm{mM}$, based on the typical concentration values used by other authors to oxidize the catechol groups) no significant changes were found on the adhesion of these films: an adhesive strength of $2.34 \pm 0.13 \mathrm{MPa}$ was obtained for the oxidized film.

Additional mechanical tests were performed in order to measure the adhesion strength of polydopamine coatings typically reported in literature, ${ }^{[20,25]}$ under the same conditions used for the developed LbL coatings. It was found that the polydopamine coatings presented a practically null adhesive strength, i.e., the glass plaques coated with polydopamine detached immediately after the beginning of the mechanical tests.

\subsection{Evaluation of Cell Behavior}

Saos-2 and L929 cells were used for the analysis of cell behavior of the developed films. These two cell lines were chosen because these are standard cell lines typically used in the biomedical field. Moreover, it is well known that cell response is quite dependent on the cell type. ${ }^{[1 b]}$ Hence, it was intended to analyse the cell behaviour of the developed coatings with cells that present distinct phenotypes, in this case Saos- 2 cells as a model for human osteoblastic cells and L929 cells to analyse fibroblasts response. The use of these cell lines allowed to analyse if the observed trends on cell response were the same or were independent of cell type.

Saos-2 and L929 cells (Figure 6A and B, respectively) were seeded on the top of CHT/HA-DN and CHT/HA 10-bilayered films. Subsequently, the biological performance of the nanostructured multilayers films was evaluated up to 21 days of culture. At predetermined time intervals, namely, $1,3,7,14$, and 21 days, the samples were compared concerning cell adhesion, viability, and proliferation. The cell response results for the glass-only control were included in Figure 6, for the sake of comparison, and they showed a good cell response as typically observed for mammalian cells when the substrate is glass.

To evaluate cell adhesion, a DAPI fluorescence assay was performed on the surfaces seeded with Saos-2 or L929 cells (Figures 6A.i-iii and 6B.i-iii, respectively). DAPI assay images show that the incorporation of DN on the multilayers led to an improved cell attachment by comparing $\mathrm{CHT} / \mathrm{HA}$ to $\mathrm{CHT} / \mathrm{HA}-\mathrm{DN}$ in both cell types. These differences are evidenced by the quantification results of the percentage of adhered cells per area presented in Figures 6A.iii and 6B. iii. The good adhesion on CHT/HA-DN films suggests that this system could have potential applicability in the development of adhesive coatings for a variety of implants requiring improved cell response. As the fabrication of these multilayered films is based on a dipping process, it would be possible to process coatings on substrates with complex geometry.

Cell viability was evaluated by alamar blue colorimetric assay. Despite of all formulations presented a good amount of well spread cells, CHT/HA-DN films also presented an enhanced cell viability for L929 and SaOs-2 cells when compared to the negative and positive controls. Specifically, for films with SaOs-2 cells, from day 3, CHT/HA-DN films present significant differences compared to CHT/ HA films (Figure 6A.iv). Additionally, only the viability of CHT/HA-DN films significantly increases with time. These results are an indicator factor that the materials employed to construct the multilayers did not jeopardize the viability of the films. Moreover, for films with L929 cells (Figure 6B. iv) besides the higher amount of cells adhered, only on day 21 were detected differences statistically significant between both multilayer films, due the lack of adhesion in the case of CHT/HA films. Ultimately, a DNA quantification assay was performed to evaluate cell proliferation. Results showed that CHT/HA-DN films had the highest proliferation for both cell types (Figure 6A.v and B.v). Additionally, these films had a continuous proliferation increase up to 14 days of culture for both cells type. Significant differences between the two multilayer films could be noticed at days 14 and 21 for both cells.

Other recent works have showed that the use of mussel adhesive proteins may contribute for a higher cell adhesion to the substrate. ${ }^{[5 e, 20]},{ }^{[25]}$ An HA-DN/Pluronic hydrogel, developed by Lee and co-workers ${ }^{[5 \mathrm{e}]}$ was subcutaneously injected into a 6-week-old mice. Both inflammatory response and fibrosis were barely visible after 21 days. This suggests that the HA-DN/Pluronic hydrogel is biocompatible and nonimmunogenic. Also, the hydrogel mass was adhered onto the nearby serous tissue of parietal peritoneum, which implied that the HA-DN/Pluronic hydrogel was adhesive to the tissue surface due to the adhesive function of unreacted catechol moieties remaining in the hydrogel structure. Ku et al. coated different substrates with polydopamine. ${ }^{[20]}$ They investigated mouse osteoblast MC3T-E1 cells adhesion on the coated substrates. When the osteoblasts were seeded onto non-adhesive polyethylene (PE), poly(tetrafluoroethylene) (PTFE), silicon rubber and poly(dimethyl-siloxane) (PDMS) substrates, the cells barely adhered and spread. On the other hand, the substrates coated with polydopamine exhibited normal adhesion. $\mathrm{Ku}$ and Park coated PCL nanofibers with polydopamine and when compared to unmodified and gelatin-coated nanofibers, human umbilical vein endothelial cells (HUVECs) exhibited highly enhanced adhesion and viability on the polydopamine coated fibers. ${ }^{[25]}$

The cell response results for polydopamine-only controls, i.e. coatings using glass as the substrate and prepared using the typical conditions adopted for these coatings ${ }^{[20,25]}$ (see experimental section), were also included in Figure 6 for the sake of comparison. As expected, these controls showed an enhanced cell response in agreement with the results of the above mentioned works. ${ }^{[20,25]}$ Therefore, the greatest advantage that the developed LbL coatings present over the typical polydopamine coatings is that they combine an enhanced cell adhesion, proliferation and viability (Figure 6) with a significantly improved adhesive strength (Figure 5), whereas the polydopamine coatings have only revealed improvements on cell response. In fact, as mentioned before, it was found that the polydopamine coatings present a practically null adhesive strength. The enhanced adhesive strength of the developed films is particularly important in specific applications, for example they can be used as coatings of a variety of implants to improve both cell response and adhesion strength in a simple and versatile way. 


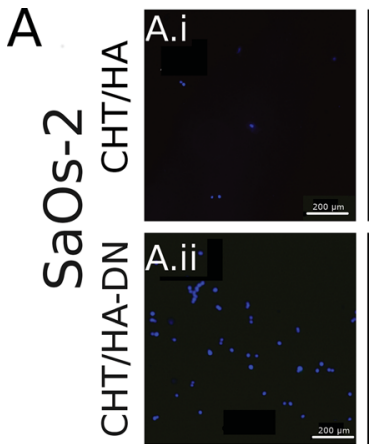

Day 1

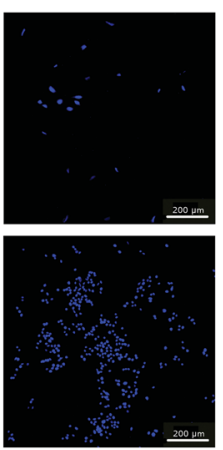

Day 3

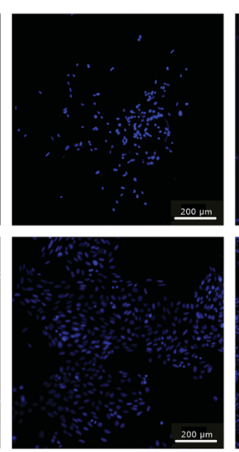

Day 7

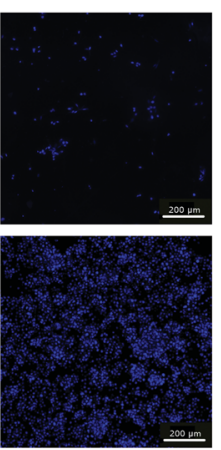

Day 14
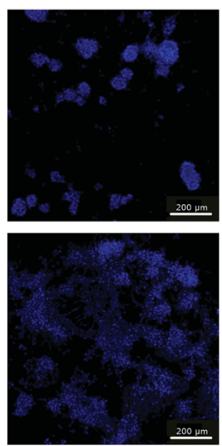

Day21

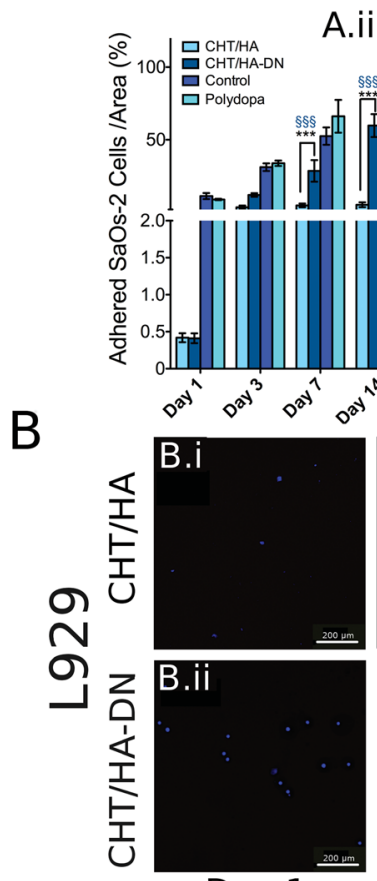

Day 1

A.iv

A.v
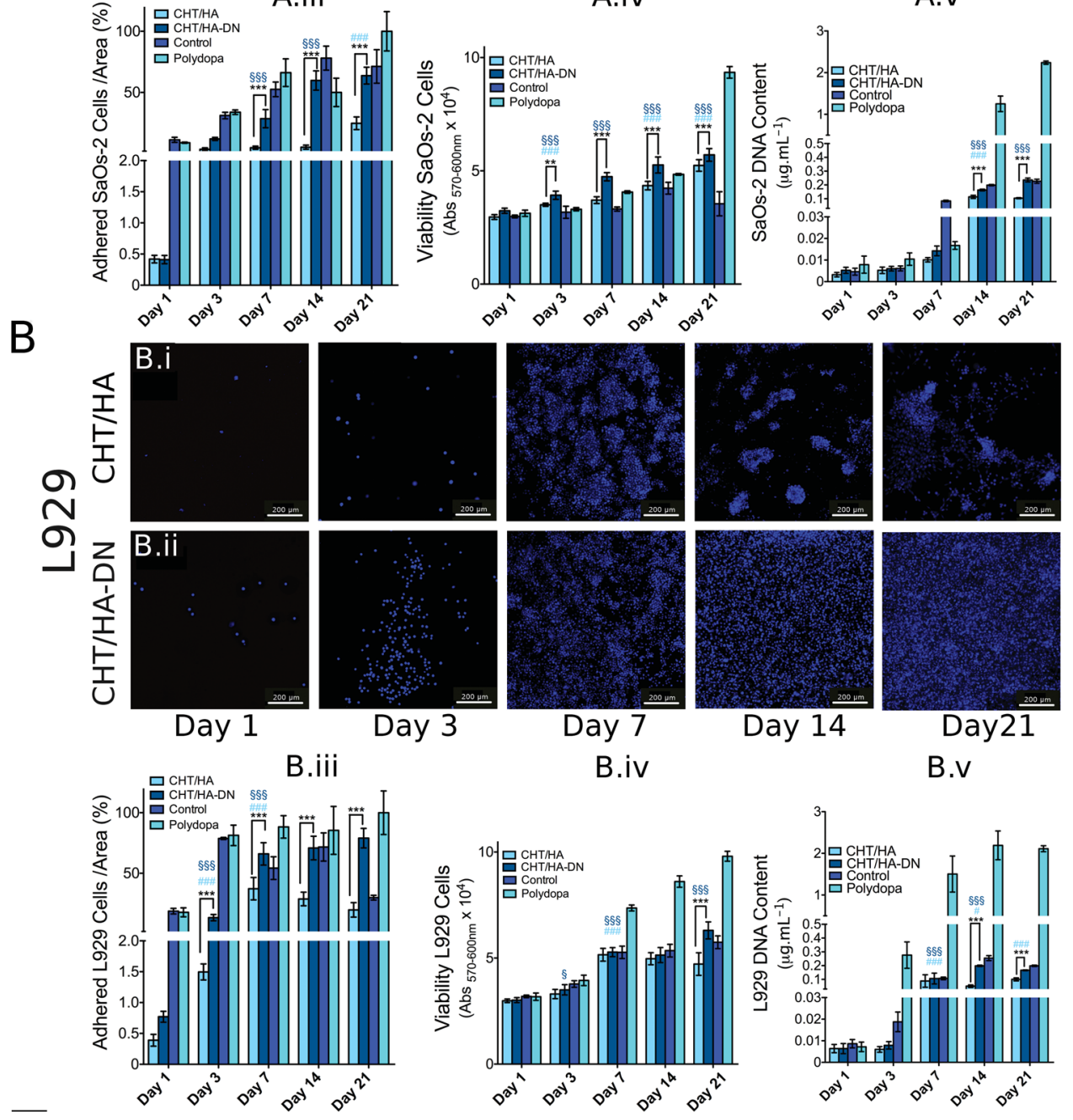

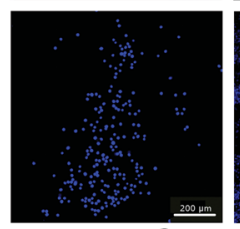

Day 3

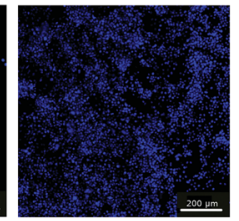

Day 7

B.iv

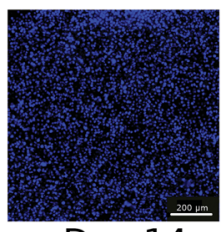

Day 14

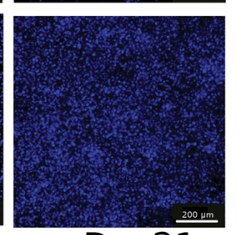

Day21

B.v
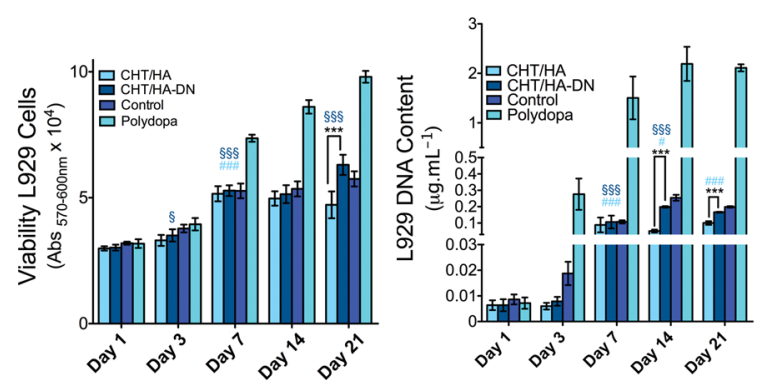

Figure 6. A) Fluorescence images of cells stained with DAPI for A) SaOs-2 and B) L929 at 1, 3, 7, 14 and 21 days of culture. Multilayer films of CHT/ HA-DN and CHT/HA (negative control), cover glasses (positive control) and polydopamine coatings were tested. Cellular density quantification was measured in the multilayer films for SaOs-2 (A.iii) and L929 (B.iii) cells. Viability of all formulations was tested for SaOs-2 (A.iv) and L929 (B.iv) cells by alamar blue. Absorbance was read at wavelength of $570 \mathrm{~nm}$ and $600 \mathrm{~nm}$. DNA quantification of all formulations was tested for SaOs-2 (A.v) and L929 (B.v) cells. Statistical differences in grouped by time point analysis were marked with $\left(^{\star}\right)$, $\left(^{\star \star}\right)$ and $\left({ }^{\star \star *}\right)$, which stand for $p$-value $<0.05$; $p<0.01$ and $p<0.001$, respectively. For evaluate the statistical differences relating to the time point before, single symbol (\# and $\S$ ) represent $p<0.05$; double symbols (\#\# and $\S$ ) $p<0.01$ and triple symbols (\#\#\# and $\S \S \S$ ) $p<0.001$. All results were presented as mean \pm standard deviation.

The increase of cell adhesion on polydopamine coated PCL fibers was attributed to the adsorption/immobilization of serum proteins on the poly-dopamine ad-layer. ${ }^{[25]}$ This layer can react with amine- or thiol-functionalized molecules via Shiff- base or Michael addition chemistry; thus, serum proteins, some of them supporting cell adhesion, can strongly attach to polydopamine coatings. Since the proteins adsorbed on polydopamine coating maintained their native structure and activity, ${ }^{[26]}$ the serum proteins attached to polydopamine layer serve as cell adhesion sites. However, as in our case 
the culture medium was not supplemented with serum, this is not the explanation for the enhanced cell adhesion on our dopamine containing LbL films. Furthermore, the mechanism behind enhanced cellular adhesion might be explained by potentially enhanced surface roughness, which could follow deposition of the polysaccharide-based films or by differences in the wettability of the surfaces. However, as discussed before, the results of Figure 4 revealed that the mechanism behind the enhanced cellular adhesion of the CHT/HA-DN films may not be explained by potentially enhanced surface roughness or by the slight difference in hydrophilicity. $\mathrm{Ku}$ et al. ${ }^{[20]}$ also found that cells adhere on polydopamine modified PTFE surfaces exhibiting well-stretched actin bundles similar to those shown in cells that adhered on normal glass substrate. The cells on polydopamine ad-layers indicate that underwent the general adhesion processes, i.e., substrate attachment /spreading/ cytoskeleton development. Moreover, it should be referred that the presence of polydopamine in the coatings proposed by other authors and in the films developed in the present work could even act as a strong anchor between cells and substrates without any covalent grafting. ${ }^{[5 c]}$ In fact the presence of the characteristic groups of DOPA and dopamine enable the formation of both covalent and non-covalent interaction. ${ }^{[5 c, 27]}$

\section{Conclusion}

HA-DN conjugates with a substitution degree of $11 \%$ were synthesized. Moreover, it was possible to successfully prepare LbL films of HA-DN and CHT. QCM-D data showed that it is possible to assemble CHT/HA-DN films. The thickness of a 10 layer film could reach nearly $75 \mathrm{~nm}$. The adhesive strength of the CHT/HA-DN films to a glass substrate was found to be significantly higher than the one of the control film. The evaluation of cell behavior demonstrated that the CHT/HA-DN films present an enhanced cell adhesion, proliferation and viability. These films could be potentially used as adhesive coatings of distinct implants in order to improve both cell response and adhesion strength in a simple and versatile way.

\section{Experimental Section}

Materials: Chitosan (CHT) with a N-deacetylation degree of $80 \%$ and a molecular weight obtained by viscosimetry of $770 \mathrm{kDa}$ was purchased from Sigma, and purified afterwards. Hyaluronic acid (HA) with a molecular weight of $595 \mathrm{kDa}$ was purchased from Sigma (ref. 53747) as hyaluronic acid sodium salt from Streptococcus equi. Dopamine hydrochloride (DN) (ref. H8502) and N-(3-Dimethylaminopropyl)$\mathrm{N}^{\prime}$-ethylcarbodiimide hydrochloride (EDC) (ref. 03450, purum, $\geq 98.0 \%$ (AT)) were purchased from Sigma and used without any further purification. Sodium periodate $\left(\mathrm{NaIO}_{4}\right)$ was purchased from Sigma (ref. 311448). Tris- $\mathrm{HCl}$ solution was purchased from Sigma (ref. 252859) as Tris (hydroxymethyl) aminomethane.
Synthesis of HA-DN: HA-DN conjugates were synthesized using EDC as an activation agent of the carboxyl groups on HA chains, based on the procedure proposed by Lee and co-workers - see Scheme 1. ${ }^{[3]}$ Briefly, $1 \mathrm{~g}$ of HA was dissolved in $100 \mathrm{~mL}$ of phosphate buffered saline (PBS) solution and the $\mathrm{pH}$ was adjusted to 5.5 with an hydrochloric acid $(\mathrm{HCl})$ aqueous solution. The solution was purged with nitrogen for 30 minutes. Then, $338 \mathrm{mg}$ of EDC and $474 \mathrm{mg}$ of DN were added and the $\mathrm{pH}$ of the reaction solution was maintained at 5.5 for 2 hours. Unreacted chemicals and urea byproducts were removed by extensive dialysis, and afterwards, the conjugate was lyophilized. In order to avoid oxidation, the conjugate was stored at $4{ }^{\circ} \mathrm{C}$ in a desiccator under vacuum and protected from the light.

Ultraviolet (UV) spectrophotometry and nuclear magnetic resonance (NMR) analysis of HA-DN: The degree of substitution of dopamine in the conjugate was determined using a UV-vis spectrophotometer (Shimadzu UV-2501 PC) and $1 \mathrm{~cm}$ quartz cells. A solution of $1 \mathrm{mg} \cdot \mathrm{mL}^{-1}$ in $0.15 \mathrm{M}$ sodium chloride $(\mathrm{NaCl})$ was prepared for the UV analysis. The samples were dissolved in deuterated water $\left(D_{2} O\right)$ for 24 hours at concentrations of $1 \mathrm{mg} \cdot \mathrm{mL}^{-1}$ and $10 \mathrm{mg} \cdot \mathrm{mL}^{-1}$, for ${ }^{1} \mathrm{H}-\mathrm{NMR}$ and ${ }^{13} \mathrm{C}-\mathrm{NMR}$ analyses respectively. The results were obtained using a spectrometer Varian Unity plus 300 and tetramethylsilane (TMS) as the internal standard. The spectra were recorded at $298 \mathrm{~K}$ and $300 \mathrm{MHz}$ for ${ }^{1} \mathrm{H}-\mathrm{NMR}$ analysis and 333 $\mathrm{K}$ and $75.4 \mathrm{MHz}$ for ${ }^{13} \mathrm{C}-\mathrm{NMR}$ analysis.

Quartz crystal microbalance (QCM-D): The formation of the multilayers of CHT and HA-DN was followed in situ by QCM-D (Q-Sense, E4 system). The mass change results from the variation of the normalized resonant frequency $(\Delta f / v)$ of an oscillating quartz crystal when adsorption occurs on the surface and the dissipation factor $(\Delta D)$ provides a measure of the energy loss in the system. If a rigid mass is adsorbed onto the surface of the piezoelectric crystal there will be a decrease in the oscillation frequency.

For viscoelastic materials the adsorbed mass does not fully couple to the oscillation of the crystal and dampens the oscillation. QCM-D allows simultaneously measuring the changes in the resonant frequency and in the viscoelastic properties (dissipation) when a film is adsorbed at the crystal surface. The measurements can be conducted at the fundamental frequency and at several overtones number $(v=1,3,5, \ldots)$.

CHT was used as the polycation while HA-DN acted as the polyanion. Fresh polyelectrolyte solutions were prepared by dissolution of $\mathrm{HA}-\mathrm{DN}$ and $\mathrm{CHT}$ in $0.15 \mathrm{M}$ of $\mathrm{NaCl}$ to yield a final concentration of $0.5 \mathrm{mg} \cdot \mathrm{mL}^{-1}$.

The sensor crystals used were AT-cut quartz (Q-Sense) with gold plated polished electrodes. These crystals were excited at $5 \mathrm{MHz}$ as well as at 15, 25, 35, 45 and $55 \mathrm{MHz}$ corresponding to the $3 \mathrm{rd}, 5 \mathrm{th}, 7 \mathrm{th}$, 9th and 11 th overtones. The crystals were previously cleaned with sequential sonication for 3 minutes in acetone,

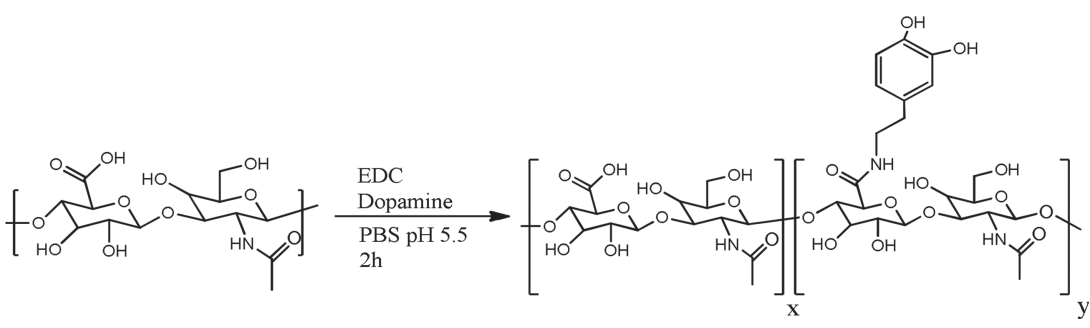

Scheme 1. Synthesis and chemical structure of HA-DN. 
ethanol and isopropanol and then dried with flowing nitrogen gas avoiding contamination prior to use. In order to ensure that the crystals are perfectly clean and therefore show a null frequency, all the experiments started with a $\mathrm{NaCl}$ baseline. Then, the polyelectrolyte solutions were injected into the cell during 10 minutes at a flow rate of $100 \mu \mathrm{L} \cdot \mathrm{min}^{-1}$ using a peristaltic pump, beginning with CHT. A rinsing step of 10 minutes with the solvent was included between the adsorptions of each polyelectrolyte. The multilayer systems were assembled at $\mathrm{pH}$ 5.5. The $\mathrm{pH}$ was adjusted with $\mathrm{HCl}$ or $\mathrm{NaOH}$. $\mathrm{CHT} / \mathrm{HA}$ films were also prepared for comparison. Films with 10 layers were produced. All experiments were conducted at $25 \stackrel{\circ}{\circ}$. During the entire process $\Delta f / v$ and $\Delta D$ shifts were continuously recorded as a function of time.

The QCM-D response of a viscoelastic film, such as the films produced in this work, can be modeled using a Voigt based mode $^{[21]}$ defined as a spring and dashpot in parallel under no slip conditions. The changes in the resonant frequency Equation (1) and in the dissipation factor Equation (2) according to Voinova and co-workers ${ }^{[21]}$ are:

$$
\begin{aligned}
& \Delta f \approx-\frac{1}{2 \pi \rho_{0} h_{0}}\left\{\frac{\eta_{B}}{\delta_{B}}+h_{L} \rho_{L} \omega-2 h_{L}\left(\frac{\eta_{B}}{\delta_{B}}\right)^{2} \frac{\eta_{L} \omega^{2}}{\mu_{L}^{2}=\omega^{2} \eta_{L}^{2}}\right\} \\
& \Delta D \approx-\frac{1}{\pi_{f} \rho_{0} h_{0}}\left\{\frac{\eta_{B}}{\delta_{B}}+2 h_{L}\left(\frac{\eta_{B}}{\delta_{B}}\right)^{2} \frac{\eta_{L} \omega}{\mu_{L}^{2}+\omega^{2} \eta_{L}^{2}}\right\}
\end{aligned}
$$

where $\omega$ is the angular frequency of the oscillation and $\rho_{0}$ and $h_{0}$ are the density and thickness of the crystal, respectively. The viscosity of the bulk liquid is $\eta_{B}, \delta_{B}\left(=2\left(\eta_{B} / \rho_{B} \omega\right)^{1 / 2}\right)$ is the viscous penetration depth of the shear wave in the bulk liquid and $\rho_{B}$ is the liquid's density. The thickness, density, viscosity and elastic shear modulus of the adsorbed layer are represented by $h_{L}, \rho_{L}, \eta_{L}$ and $\mu_{L}$ respectively.

Thickness measurements were performed using the Voigt viscoelastic model implemented in the QTools software from Q-Sense and also using an Ellipsometer equipment (SpecEl-2000-vis, Mikropack). Changes in resonant frequency and dissipation of the 7 th overtone were fitted.

Surface characterization: The surface morphology of the samples was observed using a Leica Cambridge S-360 scanning electron microscope (SEM). All samples were coated with a conductive layer of sputtered gold. The SEM micrographs were taken at an accelerating voltage of $15 \mathrm{kV}$ and at different magnifications.

The surface roughness of the samples was analyzed by atomic force microscope (AFM). AFM measurements were performed in a MultiMode STM microscope controlled by the NanoScope III from Digital Instruments system, operating in tapping mode at a frequency of $1 \mathrm{~Hz}$. At least five measurements were performed in the different sample, which had been previously air-dried.

Water contact angle measurements were also performed at room temperature using an OCA 15 plus goniometer (DataPhysics Instruments). The values were obtained by the sessile drop method. The used liquid was ultrapure water and the drop volume was $3 \mu \mathrm{L}$. At least five measurements were carried out for each sample.

Adhesion tests: The adhesion properties of the multilayer films using glass as the substrate were evaluated using a universal mechanical testing machine (Instron model 5540, USA), following the ASTM D1002 standard. Fresh polyelectrolyte solutions were prepared by dissolution of $\mathrm{HA}$ and $\mathrm{HA}-\mathrm{DN}$ in $0.15 \mathrm{M}$ of $\mathrm{NaCl}$ solution and $\mathrm{CHT}$ in $1 \%$ acetic acid, with a $\mathrm{pH} 5.5$ to yield a final concentration of $0.5 \mathrm{mg} \cdot \mathrm{mL}^{-1}$.

Glass plaques with $3 \mathrm{~mm}$ of thickness were dipped alternately in the polyelectrolyte solutions during 10 minutes, beginning with $\mathrm{CHT}$. A rinsing step of 5 minutes with $0.15 \mathrm{M} \mathrm{NaCl}$ solution was included between the adsorptions of each polyelectrolyte. Films with 10 bilayers were produced onto the surface of each glass plaque. Then, these films were washed until a neutral $\mathrm{pH}$ was achieved, in order to perform the mechanical tests at the same $\mathrm{pH}$ as the biological tests. Additionally, glass plaques coated with oxidized films were prepared by immersion in a $5 \mathrm{mM}$ sodium periodate solution during 2 hours, after the procedure previously described. The oxidized agent and oxidation time were chosen based on literature. ${ }^{[24]}$ In fact, sodium periodate is an effective oxidizing agent of the catechol groups.

Glass plaques with a polydopamine coating were also prepared using the procedure described by Lee $\mathrm{H}$. et al. ${ }^{[5 \mathrm{c}]}$ Briefly, the glass slides were immersed into a dopamine solution (2 $\mathrm{mg} \cdot \mathrm{mL}^{-1}$ in $10 \mathrm{mM}$ tris- $\left.\mathrm{HCl}, \mathrm{pH} 8.5\right)$ at $25^{\circ} \mathrm{C}$. $\mathrm{pH}$-induced oxidation changes the solution color to dark brown. The substrates were immersed in the polydopamine solution overnight. Then, the coated surfaces were rinsed with ultrapure water and dried at room temperature.

All the adhesion experiments were conducted at $25^{\circ} \mathrm{C}$. A pair of plates was put in contact and covered areas with $10 \times 20 \mathrm{~mm}^{2}$ were superimposed and maintained at $40{ }^{\circ} \mathrm{C}$ overnight. Then, the bonded glass slides were placed on the testing machine and $a$ crosshead speed of $5 \mathrm{~mm} . \mathrm{min}^{-1}$ was employed. The samples were stressed until enough force was applied to trigger their detachment and pull them apart. The lap shear bonding strength was then determined from the maximum of the force-deformation curve obtained. The average and standard deviations were determined using the results from five samples.

Evaluation of cell behavior: Two cell lines, namely, a mouse lung fibroblastic cell line (L929) and a human primary osteosarcoma cell line (SaOs-2), were obtained from European Collection of cell Cultures (ECA CC, UK). The cells were cultured with low glucose DMEM supplemented with $3.7 \mathrm{mg} \cdot \mathrm{mL}^{-1}$ sodium bicarbonate, 10\% FBS, and $1 \%$ penicillin-streptomycin at $\mathrm{pH} 7.4$. The cells were grown in $75 \mathrm{~cm}^{2}$ tissue culture flasks and incubated at $37{ }^{\circ} \mathrm{C}$ in a humidified air atmosphere of $5 \% \mathrm{CO}_{2}$. The medium was changed every 3-4 days. At $90 \%$ of confluence, cells grown in tissue culture flasks were washed with PBS and subsequently detached by a chemical procedure with $0.05 \%$ trypsin-EDTA solution for $5 \mathrm{~min}$ at $37{ }^{\circ} \mathrm{C}$ in a humidified air atmosphere of $5 \% \mathrm{CO}_{2}$. To inactivate the trypsin effect, cell culture medium was added. The cells were then centrifuged at $300 \mathrm{~g}$ and $25^{\circ} \mathrm{C}$ for $5 \mathrm{~min}$ and the medium was decanted.

Prior to cell seeding, the samples were sterilized by UV radiation for $1 \mathrm{~h} .30 \mu \mathrm{L}$ of supplemented DMEM containing a cell suspension with $5 \times 10^{3}$ cells was added to a 10 bilayered CHT/HA-DN film, the respective negative control $\mathrm{CHT} / \mathrm{HA}$, the positive control (cover glasses) and polydopamine coated cover glasses, also used as a control (in triplicate). The polydopamine coating was performed adopting the same conditions used for the mechanical adhesion tests. Then, the samples were incubated at $37{ }^{\circ} \mathrm{C}$ in a humidified air atmosphere of $5 \% \mathrm{CO}_{2}$. After 4 hours supplemented DMEM was added.

To obtain fluorescent images, DAPI fluorescent assay was performed at each time culture period. DAPI stains preferentially 
double-stranded DNA by delineating cells nuclei in blue. Prior to staining, at each time point, the culture medium was removed and $10 \%$ formalin was added to each well (in triplicate). After 1 hour at room temperature, formalin was removed and the samples were washed with phosphate buffer saline (PBS). $1 \mathrm{~mL}$ of PBS containing $1 \mu \mathrm{L}$ of DAPI was added to each well. After 1 hour at room temperature protected from light, samples were washed three times with PBS and immediately visualized in the dark by fluorescence microscopy. ImageJ software was used to quantify the cells adhered on the multilayer films.

The samples were tested for cytotoxicity using alamarBlue colorimetric assay. Briefly, the samples with adhered cells were placed in a non-treated surface 24-well cell culture plate (in triplicate) and incubated at $37{ }^{\circ} \mathrm{C}$ and $5 \% \mathrm{CO}_{2}$. At 1, 3, 7, 14 and 21 days of culture, the assay was performed, protected from light. The culture medium was removed and $500 \mu \mathrm{L}$ of supplemented DMEM containing alamarBlue solution with a dilution ratio of $1: 10$ was added to each well. The samples were then incubated in the dark at $37{ }^{\circ} \mathrm{C}$ and $5 \% \mathrm{CO}_{2}$. After $3 \mathrm{~h}, 100 \mu \mathrm{L}$ of each well (in triplicate) was transferred to a 96-well plate. The absorbance was monitored at $570 \mathrm{~nm}$ and $600 \mathrm{~nm}$ using a microplate reader (Synergy HT, Bio-TEK).

A DNA quantification assay was also performed in order to evaluate cell proliferation in the samples. For each culture time, the wells of the plate (the same samples used in viability assay) were extensively washed with PBS, and then, $1 \mathrm{~mL}$ of ultrapure sterile water was added to each well. The well plate was placed in a shaking water bath at $37{ }^{\circ} \mathrm{C}$ for 1 hour. Ultimately, the plates were immediately stored at $-80^{\circ} \mathrm{C}$ until use. The quantification of total DNA was determined after cell lysis, according to the manufacturer's description. After transferring each solution to a 96-well white opaque plate (in triplicates), the plate was incubated at room temperature protected from light for 10 minutes. The standard curve for DNA analysis was generated with provided DNA from the assay kit. Fluorescence was read at excitation of $485 / 20 \mathrm{~nm}$ and emission of $528 / 20$ nm using a microplate reader (Synergy HT, BioTek, U.S.A.).

Statistical Analysis was performed to analyze significant differences between formulations. Data of viability and DNA were grouped by formulation, namely, Control (positive control), polydopa, CHT/HA-DN, and CHT/HA (negative control), to assess time-course significant differences of each formulation. Statistical analysis of both data cases was performed using two-way analysis of variance (ANOVA) with Bonferroni post-test using GraphPad Prism 5.0 software. The adopted nomenclature was the following: statistical differences in grouped by time point analysis were marked with $(*),\left({ }^{\star *}\right)$ and $\left({ }^{\star *}\right)$, which stand for $p$-value $<0.05$; $p<0.01$ and $p<0.001$, respectively. For evaluate the statistical differences relating to the time point before, symbols as \# and $\S$ were used to represent $\mathrm{CHT} / \mathrm{HA}$ and $\mathrm{CHT} / \mathrm{HA}-\mathrm{DN}$ groups respectively. Single symbol (\# and $\S$ ) represent $p<0.05$; double symbols (\#\# and§§) $p<0.01$ and triple symbols (\#\#\# and $\S \S \S) ~ p<0.001$. All results were presented as mean \pm standard deviation.

\section{Acknowledgements}

The authors want to acknowledge the COST Action TD0906 - Biological adhesives: from biology to biomimetics. The authors also acknowledge the financial support from the Fundação para a Ciência e para a Tecnologia through the Ph.D. grants with the references SFRH/BD/73119/2010 and SFRH/BD/69529/2010. G.G. Ferrer acknowledges the support of the Spanish Ministry of Science and Innovation for the mobility grant JC2008-00135. G. Botelho acknowledges the NMR portuguese network (PTNMR, Bruker Avance III 400-Univ. Minho).

[1] a) Z. Ma, Z. Mao, C. Gao, Colloids Surf., B 2007, 60, 137; b) N. M. Alves, I. Pashkuleva, R. L. Reis, J. F. Mano, Small 2010, 6, 2208.

[2] H. Shin, S. Jo, A. G. Mikos, Biomaterials 2003, 24, 4353.

[3] H. Lee, Y. Lee, A. R. Statz, J. Rho, T. G. Park, P. B. Messersmith, Adv. Mater. 2008, 20, 1619.

[4] a) J. H. Waite, M. L. Tanzer, Science 1981, 212, 1038; b) Y. Lee, H. Lee, Y. B. Kim, J. Kim, T. Hyeon, H. Park, P. B. Messersmith, T. G. Park, Adv. Mater. 2008, 20, 4154; c) A. I. Neto, H. J. Meredith, C. L. Jenkins, J. J. Wilker, J. F. Mano, Rsc Adv 2013, 3, 9352.

[5] a) M. E. Yu, T. J. Deming, Macromolecules 1998, 31, 4739; b) T. J. Deming, Curr Opin Chem Biol 1999, 3, 100; c) H. Lee, S. M. Dellatore, W. M. Miller, P. B. Messersmith, Science 2007, 318, 426; d) T. H. Anderson, J. Yu, A. Estrada, M. U. Hammer, J. H. Waite, J. N. Israelachvili, Adv. Funct. Mater. 2010, 20, 4196; e) Y. Lee, H. J. Chung, S. Yeo, C. H. Ahn, H. Lee, P. B. Messersmith, T. G. Park, Soft Matter 2010, 6, 977.

[6] a) T. Serizawa, M. Yamaguchi, T. Matsuyama, M. Akashi, Biomacromolecules 2000, 1, 306; b) C. Picart, P. Lavalle, P. Hubert, F. J. G. Cuisinier, G. Decher, P. Schaaf, J. C. Voegel, Langmuir 2001, 17, 7414; c) X. Qiu, S. Leporatti, E. Donath, H. Möhwald, Langmuir 2001, 17, 5375; d) Y. Liu, T. He, C. Gao, Colloids Surf., B 2005, 46, 117; e) J. Zhang, B. Senger, D. Vautier, C. Picart, P. Schaaf, J. C. Voegel, P. Lavalle, Biomaterials 2005, 26, 3353; f) A. Schneider, C. Vodouhe, L. Richert, G. Francius, E. Le Guen, P. Schaaf, J. C. Voegel, B. Frisch, C. Picart, Biomacromolecules 2007, 8, 139; g) C. R. Wittmer, J. A. Phelps, W. M. Saltzman, P. R. Van Tassel, Biomaterials 2007, 28, 851; h) Z. R. Wu, J. Ma, B. F. Liu, Q. Y. Xu, F. Z. Cui, J. Biomed. Mater. Res., Part A 2007, 81A, 355.

[7] J. R. E. Fraser, T. C. Laurent, U. B. G. Laurent, J Intern Med 1997, 242, 27.

[8] W. G. Pitt, R. N. Morris, M. L. Mason, M. W. Hall, Y. Luo, G. D. Prestwich, J. Biomed. Mater. Res., Part A 2004, 68A, 95.

[9] L. Lapcik, L. Lapcik, S. De Smedt, J. Demeester, P. Chabrecek, Chem. Rev. 1998, 98, 2663.

[10] P. H. Chua, K. G. Neoh, E. T. Kang, W. Wang, Biomaterials 2008 , 29, 1412.

[11] a) M. Rinaudo, M. Milas, P. Ledung, Int J Biol Macromol 1993, 15, 281; b) L. L. Hench, Biomaterials 1998, 19, 1419; c) R. Jayakumar, M. Prabaharan, R. L. Reis, J. F. Mano, Carbohydr. Polym. 2005, 62, 142.

[12] a) A. Charlot, V. Sciannaméa, S. Lenoir, E. Faure, R. Jérôme, C. Jérôme, C. Van De Weerdt, J. Martial, C. Archambeau, N. Willet, A.-S. Duwez, C.-A. Fustin, C. Detrembleur, J. Mater. Chem. 2009, 19, 4117; b) X. M. Zhang, Z. Y. Li, X. B. Yuan, Z. D. Cui, X. J. Yang, Appl. Surf. Sci. 2013, 284, 732.

[13] a) J. Baier Leach, K. A. Bivens, C. W. Patrick Jr. , C. E. Schmidt, Biotechnol. Bioeng. 2003, 82, 578; b) T. Segura, B. C. Anderson, P. H. Chung, R. E. Webber, K. R. Shull, L. D. Shea, Biomaterials 2005, 26, 359; c) Z. Bezáková, M. Hermannová, E. Dřímalová, A. Malovíková, A. Ebringerová, V. Velebný, Carbohydr. Polym. 2008, 73, 640 .

[14] D. D. Mueller, T. D. Morgan, J. D. Wassenberg, T. L. Hopkins, K. J. Kramer, Bioconjugate Chem. 1993, 4, 47.

[15] Y. Tokita, A. Okamoto, Polym. Degrad. Stabil. 1995, 48, 269. 
[16] a) B. J. Kvam, M. Atzori, R. Toffanin, S. Paoletti, F. Biviano, Carbohydr. Res. 1992, 230, 1; b) A. J. Metta-Magana, R. Reyes-Martinez, H. Tlahuext, Carbohydr. Res. 2007, 342, 243.

[17] S.-M. Chen, K.-T. Peng, J. Electroanal. Chem. 2003, 547, 179.

[18] a) L. Richert, P. Lavalle, E. Payan, X. Z. Shu, G. D. Prestwich, J. F. Stoltz, P. Schaaf, J. C. Voegel, C. Picart, Langmuir 2004, 20, 448; b) O. Etienne, A. Schneider, C. Taddei, L. Richert, P. Schaaf, J. C. Voegel, C. Egles, C. Picart, Biomacromolecules 2005, 6, 726.

[19] a) J. Ruths, F. Essler, G. Decher, H. Riegler, Langmuir 2000, 16, 8871 ; b) R. A. McAloney, M. Sinyor, V. Dudnik, M. C. Goh, Langmuir 2001, 17, 6655.

[20] S. H. Ku, J. Ryu, S. K. Hong, H. Lee, C. B. Park, Biomaterials 2010 31, 2535.

[21] M. V. Voinova, M. Rodahl, M. Jonson, B. Kasemo, Phys. Scripta 1999, 59, 391.
[22] a) M. Edvardsson, S. Svedhem, G. Wang, R. Richter, M. Rodahl, B. Kasemo, Anal. Chem. 2009, 81, 349; b) G. V. Martins, J. F. Mano, N. M. Alves, Carbohydr. Polym. 2010, 80, 570; c) G. V. Martins, E. G. Merino, J. F. Mano, N. M. Alves, Macromol. Biosci. 2010, 10, 1444.

[23] K. Yamada, T. H. Chen, G. Kumar, O. Vesnovsky, L. D. T. Topoleski, G. F. Payne, Biomacromolecules 2000, 1, 252.

[24] B. P. Lee, J. L. Dalsin, P. B. Messersmith, Biomacromolecules 2002, 3, 1038.

[25] S. H. Ku, C. B. Park, Biomaterials 2010, 31, 9431.

[26] H. Lee, J. Rho, P. B. Messersmith, Adv. Mater. 2009, 21, 431.

[27] H. Lee, N. F. Scherer, P. B. Messersmith, Proc. Natl. Acad. Sci. U. S. A. 2006, 103, 12999.

Received: November 18, 2013 Revised: February 7, 2014 Published online: 